\title{
$\infty$ \\ Comprometimento Neurocognitivo em Modelos Experimentais Murinos: Aplicação para o Estudo dos Déficits Associados à Malária Não Grave
}

\author{
Beatriz Nogueira Siqueira e Silva, Pamela Rosa Gonçalves, Aline Barbosa Maia, \\ Caroline Cristhiani Tavares de Lima Gress, Rachel Mazzei Moura de Andrade Lins, \\ Luciana Pereira de Sousa \& Cláudio Tadeu Daniel-Ribeiro \\ Laboratório de Pesquisa em Malária, Instituto Oswaldo Cruz, Centro de Pesquisa, Diagnóstico \\ e Treinamento (CPD-Mal), Fiocruz. Ministério da Saúde
}

\section{Resumo}

Usualmente, a malária se manifesta como uma tríade clássica de sintomas, podendo evoluir para formas graves, sendo a malária cerebral a mais letal das complicaçôes da doença causada pelo Plasmodium falciparum. Pesquisas em modelos experimentais ajudam a compreender os diversos mecanismos de evolução, tratamentos, aspectos genéticos e moleculares e déficits cognitivo-comportamentais associados à malária cerebral. Nossa equipe vem desenvolvendo, no Laboratório de Pesquisa em Malária do Instituto Oswaldo Cruz, Fiocruz (LPM, IOC, Fiocruz), estudos sobre o efeito de estímulos imunes no comportamento animal, com foco no comprometimento da memória e na manifestaçáo de ansiedade, relatados como associados mesmo a formas não graves de malária em humanos. A estratégia adotada, o uso de modelos experimentais murinos suscetíveis ao desenvolvimento de malária cerebral, os estudos em perspectiva pelo nosso grupo e outros aspectos relacionados são abordados nesse breve sobrevoo da literatura específica recente.

Palavras-chave: comportamento, déficits cognitivos, malária, modelo murino, neurocognição, parâmetros cognitivo-comportamentais.

\section{Abstract}

Malaria usually manifests as a classic triad of symptoms that can evolve to severe forms; cerebral malaria being the most lethal of the complications of the disease 
caused by Plasmodium falciparum. Research in experimental models helps to understand the different evolution mechanisms, treatments, genetic and molecular aspects and cognitive-behavioral deficits associated with cerebral malaria. Our team has been conducing, at the Laboratório de Pesquisa em Malária do Instituto Oswaldo Cruz, Fiocruz (LPM, IOC, Fiocruz), studies on the effect of immune stimuli on animal behavior, focusing on memory impairment and on the manifestation of anxiety, reported as events associated even with the non-severe forms of malaria in humans. The adopted strategy, the use of experimental murine models susceptible to the development of cerebral malaria, the planned studies and other related aspects focused by our group are addressed in this brief overview of recent specific literature.

Keywords: behavior, cognitive deficits, malaria, murine model, neurocognition, cognitive-behavioral parameters.

\section{INTRODUÇÃo}

A malária é uma doença infecto-parasitária causada por protozoários do gênero Plasmodium, sendoo $P$. falciparum a espécie de maior prevalência no mundo, especialmente na África, onde afeta, de forma mais grave, crianças de até 5 anos de idade. Em 2019, foram relatados 229 milhóes de casos e 409 mil mortes devidos à doença no mundo (1), o que faz dela um dos principais fardos de saúde pública do mundo. Somam-se a esses dados o elevado sofrimento que impóe às suas vítimas e o colossal impacto econômico nas naçóes afetadas (com gastos em açóes de prevenção, controle e tratamento, além das perdas decorrentes das vidas ceifadas, dos dias não trabalhados durante a doença aguda e das sequelas neurológicas, cognitivas e comportamentais temporárias ou de longo prazo). A transmissão é garantida pelo vetor, o mosquito fêmea do gênero Anopheles, e oito espécies do Plasmodium: P. falciparum, P. vivax, P. malariae, P. ovale curtisi, $P$. ovale wallikeri, P. knowlesi, P. cynomolgi e P. simium podem causar doença no homem (2). O P. falciparum é a espécie responsável pela maioria dos casos e dos óbitos e pela malária cerebral (MC), a forma mais grave da parasitose. Segundo a OMS, em 2017, essa espécie foi responsável por 99,7\% dos casos de malária na região africana, 62,8\% no Sudeste Asiático, 69\% no Mediterrâneo Oriental e 71,9\% no Pacífico Ocidental (3).

A febre, o calafrio intenso e a sudorese compóem a tríade clássica de sintomas da doença, que é frequentemente associada à cefaleia intensa. A malária pode evoluir para formas graves em decorrência de processos patológicos, incluindo uma resposta imune exagerada à infecção plasmodial (4).

A malária cerebral (MC) acomete 1 a 2\% 
dos casos de malária por $P$. falciparum (1). Sob o ponto de vista clínico, ela se apresenta de forma variada com sinais e sintomas que podem simular encefalites virais, meningoencefalites de origem bacteriana ou fúngica, insolação, febre tifoide cerebral, abscesso cerebral, eventos cerebrovasculares, intoxicaçóes, epilepsia, dentre outras (5). Modelos experimentais murinos apresentam sinais semelhantes ao da malária humana, como ataxia, paralisia, coma e convulsões (5-7).

Algumas descobertas importantes que ajudaram a compreensão da patogênese da malária feitas em modelos murinos ao longo dos anos foram: a) micro-hemorragias, edema e obstrução de vasos por células mononucleares no cérebro caracterizam-se como sinais de MC experimental (8,9); b) algumas linhagens de camundongo podem ser resistentes ao desenvolvimento de $\mathrm{MC}$ e ir a óbito devido à hiperparasitemia, envolvendo fatores genéticos à suscetibilidade para o desenvolvimento da MC (10-13); c) para o surgimento de sinais neurológicos reveladores de comprometimento cerebral na malária é necessário o rompimento da barreira hematoencefálica (BHE) $(11,13)$ e a sua permeabilidade se associa à hemorragia, oclusão de vasos capilares por leucócitos (modelo murino C57BL/6) ou por glóbulos vermelhos parasitados (humanos); d) o extravasamento vascular (principal evento patológico durante a $\mathrm{MC}$ ) e subsequente edema são maiores durante a $\mathrm{MC}$ experimental por Plasmodium berghei ANKA (PbA) e na MC humana, do que nas respectivas formas náo complicadas da doença (14). Além da descoberta de diversos mecanismos patogenéticos da $\mathrm{MC}$, os modos de reversáo ou mitigação dos danos causados pela doença também foram bastante explorados: i) o tratamento com óxido nítrico, protegendo de hemorragias cerebrais e inflamação (15); ii) a terapia adjuvante com células do estroma mesenquimal na proteção de danos vasculares e melhora do comportamento semelhante à depressáo causados pela $\mathrm{MC}$ experimental (16); iii) e o tratamento com extrato etanólico proveniente do fungo Trichoderma stromaticum atuando na prevenção de sinais neurológicos em modelo suscetível à MC (17), entre outros.

Sabe-se que sobreviventes de MC podem apresentar sequelas neurocognitivas, como déficits motores, de aprendizado, e outras a curto ou longo prazo após as crises febris (18). Tais déficits também têm sido relatados em modelos experimentais murinos de MC (19,20). Nos últimos anos, mau desempenho em eventos cognitivos, principalmente relacionados à aprendizagem e memória, também foi observado, como resultado de malária não grave $(\mathrm{MnG}) \mathrm{em}$ humanos em várias regióes endêmicas do mundo $(21,22)$, mas não o haviam sido em alguns modelos experimentais murinos tradicionais de $\mathrm{MnG}$ (23) até recentemente 
(24), sugerindo que o surgimento de sequelas neurocognitivas parece depender da interação parasita-hospedeiro, envolvendo aspectos genéticos do hospedeiro e das espécies e cepas parasitárias, assim como da natureza e intensidade da resposta inflamatória.

Células do sistema imune estão presentes nos compartimentos cerebrais e podem desempenhar importante papel no sistema nervoso central. As citocinas podem afetar os processos cognitivos, como aprendizagem e memória, o comportamento social e psíquico, além de estarem relacionadas a doenças neurodegenerativas (25). O conhecimento de seus papéis pode ajudar a compreensão da relação entre os sistemas imune e nervoso na homeostase, em processos crônico degenerativos e em doenças infecciosas ou parasitárias, como a malária. $\mathrm{O}$ uso de modelos experimentais murinos é importante para essa compreensão, já que o estudo em humanos é limitado ao post-mortem pelas óbvias restriçôes éticas e legais (26). Embora a malária experimental murina não reproduza exatamente a malária humana, ela apresenta similaridade considerável em relação à resposta imune e aspectos clínicos, o que é importante para cientistas confirmarem, ajustarem ou excluírem hipóteses (26). Diferentes linhagens de camundongos apresentam diferentes suscetibilidades ao desenvolvimento da MC experimental, de acordo com a espécie ou cepa de Plasmodium.
Tabela 1. Suscetibilidade ao desenvolvimento de $\mathrm{MC}$ de acordo com a linhagem de camundongo e as diferentes espécies/cepas de Plasmodium.

\begin{tabular}{|c|c|c|c|}
\hline $\begin{array}{l}\text { Espécies de } \\
\text { Plasmodium }\end{array}$ & $\begin{array}{l}\text { Linhagens de } \\
\text { camundongo }\end{array}$ & $\begin{array}{l}\text { Malária } \\
\text { Cerebral }\end{array}$ & Referências \\
\hline $\begin{array}{l}\text { Plasmodium } \\
\text { berghei } K 173\end{array}$ & C57BL/6 & + & (27) \\
\hline $\begin{array}{l}\text { Plasmodium } \\
\text { berghei ANKA }\end{array}$ & $\begin{array}{l}\text { CBA/ca } \\
\text { BALB/c } \\
\text { C57BL/6 } \\
\text { DBA/2 } \\
\text { Swiss }\end{array}$ & $\begin{array}{l}+ \\
- \\
+ \\
+ \\
+\end{array}$ & $\begin{array}{l}(28) \\
(29) \\
(30) \\
(5)\end{array}$ \\
\hline \multirow{2}{*}{$\begin{array}{l}\text { Plasmodium } \\
\text { berghei NK65 }\end{array}$} & WM/Ms & + & (31) \\
\hline & C57BL/6 & - & (32) \\
\hline $\begin{array}{l}\text { Plasmodium } \\
\text { yoelli 17XL }\end{array}$ & $\begin{array}{l}\text { BALB/c } \\
\text { DBA2 } \\
\text { Swiss }\end{array}$ & $\begin{array}{l}+ \\
- \\
+\end{array}$ & $\begin{array}{l}(33) \\
(33) \\
(34)\end{array}$ \\
\hline $\begin{array}{l}\text { Plasmodium } \\
\text { chabaudi } \\
\text { chabaudi }\end{array}$ & $\begin{array}{l}\text { C57BL/6 } \\
\text { CBA/J } \\
\text { BALB/c }\end{array}$ & $\begin{array}{l}- \\
- \\
-\end{array}$ & $\begin{array}{l}(26) \\
(26) \\
(26)\end{array}$ \\
\hline $\begin{array}{l}\text { Plasmodium } \\
\text { chabaudi } \\
\text { adami }\end{array}$ & C57BL/6 & - & (35) \\
\hline
\end{tabular}

Apesar de décadas de pesquisa sobre a fisiopatogenia da malária, há muito a ser descoberto e entendido e a utilização do modelo murino é ainda indispensável para o avanço do conhecimento sobre as complicaçóes da doença. Como a grande maioria dos casos de malária humana se apresenta como MnG por P. falciparum (4), o nosso grupo no LPM, IOC-Fiocruz usou um modelo experimental murino que julgamos simular o curso clínico da malária falciparum, que tem o potencial de se agravar e evoluir para $\mathrm{MC}$, se não tratada oportunamente. Assim, camundongos C57BL/6 foram infectados por $\mathrm{PbA}$, mas tratados no $4^{\circ}$ dia após a 
infecção, antes do aparecimento de qualquer sinal clínico de MC, que ocorre por volta do $6^{\circ}$ dia (36), para responder às seguintes perguntas:

i) Qual a influência da MnG em paradigmas comportamentais associados aos parâmetros de memória e ansiedade em um modelo murino com capacidade de desenvolver malária cerebral?

A partir do protocolo citado acima, 83 dias após o tratamento e cura radical da infecção, os animais foram submetidos a testes comportamentais, com a finalidade de analisar o desempenho da memória de reconhecimento e de comportamento do tipo ansioso em tarefas como o campo aberto (Open Field Test - OFT); visando avaliar mobilidade, memória de habituaçáo e ansiedade; o teste de reconhecimento de objeto novo (Novel Object Recognition Test - NORT), para mensurar especificamente memória de longa duração; e o teste claro/ escuro para mensuração específica do comportamento ansioso. Nosso grupo observou que a infecçáo por $P b A$ pode causar déficits de memória e induzir comportamento do tipo ansioso detectável 83 dias após o término do tratamento de episódio único de $\mathrm{MnG}$ (24, dados ainda náo publicados sugerem que as alteraçoóes detectadas tardiamente são persistentes, 37). Nossos dados, analisados à luz de parâmetros de longevidade (38), sugerem que sequelas cognitivas poderiam ser detectáveis em humanos sete (a 12) anos após um episódio de MnG.

\section{ii) Qual é a influência de estímulos imunes} nas sequelas cognitivas resultantes da malária experimental não grave?

Sabe-se que diferentes padróes de resposta imune são desencadeados por estímulos distintos e podem induzir ou influenciar habilidades e comportamentos cognitivos humanos diversos (39). Um trabalho recente de nosso grupo avaliou também esse aspecto (40). Duas semanas após o tratamento (protocolo acima), iniciam-se as imunizaçóes com as seguintes vacinas e antígenos: vacina antidiftérica e antitetânica (dT) para adultos, vacina contra influenza, Proteína 3 de superfície de merozoíto de Plasmodium falciparum (proteína recombinante $P f$ MSP-3 e vacina experimental para a malária), ovalbumina de ovo de galinha branca (OVA) e lipopolissacarídeo de Escherichia coli (EcLPS). Para a imunização usaram-se: a) todos os antígenos e vacinas descritas acima (grupo experimental Pool); b) a combinação de antígeno e vacina ( $E c$ LPS e Influenza) indutoras do padráo de resposta imune (RI) do tipo 1 (Th1); e c) antígenos e vacinas (OVA, vacina $\mathrm{dT}$ e a Pf MSP-3) indutores de RI do tipo 2 (Th2). Os estímulos anti-inflamatórios (Th2) aprimoraram a memória de longa duração de camundongos sadios adultos e evitaram a manifestação do déficit comportamental detectado tardiamente nos camun- 
dongos com episódio prévio de $\mathrm{MnG}$ por PbA, sugerindo um efeito promotor benéfico da imunização no desempenho cognitivo-comportamental e abrindo novas possibilidades para a utilização de vacinas, além do uso clássico como profiláticos de doenças infecciosas (40).

iii) Qual é a dinâmica das alteraçóes cognitivas e comportamentais da $\mathrm{MnG}$ e o efeito da imunomodulação com um estímulo imune isolado?

Déficits cognitivos e alteraçóes comportamentais estão associados à $\mathrm{MC}$ e mesmo à $\mathrm{MnG}$, contudo não há tratamento efetivo para tais comprometimentos. Considerando o efeito de estímulos imunes integrados, do perfil de resposta $T h 2$, na recuperação de déficits cognitivos e fenótipo do tipo ansioso, detectados tardiamente na $\mathrm{MnG}$ em modelo suscetível ao desenvolvimento de MC (40), buscou-se avaliar a dinâmica das alterações após MnG associada ao estudo das propriedades imunomoduladoras de apenas um imunógeno, na reversão dos danos na MnG por PbA. Resultados ainda não publicados, indicam que os danos cognitivos e as alterações comportamentais da $\mathrm{MnG}$ são detectáveis logo após o tratamento com o antimalárico cloroquina e podem persistir por prazos ainda maiores aos detectados anteriormente. Os dados preliminares também sugerem que o tratamento com um só imunógeno indutor de resposta imune de perfil Th2 é capaz de reverter danos cogni- tivos e comportamentais (37) confirmando que estímulos imunes são uma abordagem promissora para reverter a manifestação de déficits cognitivos e alteraçóes comportamentais da MnG.

iv) Qual a influência da $\mathrm{MnG}$ em parâmetros de memória e ansiedade em um modelo murino sem capacidade de desenvolver malária cerebral?

Mundialmente, as espécies causadoras de malária humana mais comuns são $P$. falciparum e $P$. vivax. Apesar de sua menor prevalência, é sabido que a infecção por $P$. vivax pode acarretar déficits cognitivos, embora raramente progrida para a malária cerebral $(22,41)$. Os modelos experimentais murinos clássicos que reproduzem déficits cognitivos da malária são modelos suscetíveis ao desenvolvimento de MC experimental. Baseados nessa conjuntura, emergiu a necessidade de estudar déficits cognitivos em modelo experimental insuscetível ao desenvolvimento de MC, embora sujeito a sinais de inflamação na microvasculatura cerebral com o modelo C57BL/6 infectado com a cepa NK65 de P. berghei (PbNK65) (32). Nosso racional é o de que um estudo comparativo com a cepa ANKA de $P$. berghei, sensível às complicaçóes da $\mathrm{MC}$ em camundongos $\mathrm{C} 57 \mathrm{BL} / 6$, pode contribuir para a compreensão de mecanismos envolvidos na gênese dos déficits cognitivos da $\mathrm{MnG}$. O nosso grupo tem trabalhado no 
estabelecimento do modelo e já fez avanços na determinação do inóculo de PbNK65 capaz de gerar parasitemias comparáveis àquelas causadas pelo $\mathrm{PbA}$, embora ainda precisemos definir o tratamento para a cura radical da infecção por PbNK65 antes de aplicar o modelo ao estudo de alteraçóes cognitivo-associados à MnG.

v) Podem os exercícios físicos atuar de forma benéfica nas funçôes cognitivas na homeostase ou sobre as sequelas cognitivas da malária experimental náo grave?

O exercício físico regular promove uma série de respostas e adaptações fisiológicas que dependem do volume e intensidade da atividade (42, 43). Alterações metabólicas promovidas pelo exercício físico têm sido associadas à modulação do sistema imune (44) podendo influenciar a resposta imune do hospedeiro a agentes infecciosos (45). A prática de exercícios físicos, pode influenciar a resposta do sistema nervoso e corresponder a uma possível estratégia aperfeiçoadora da função neurocognitiva, em humanos sadios: memória de trabalho (46) e função executiva (atenção, inteligência fluida, planejamento e raciocínio lógico, 47, 48), ou em doenças neurodegenerativas (performance cognitiva na esclerose múltipla, 49); além da memória de longa duração em ratos sadios e epilépticos (50) e em modelo experimental de Alzheimer (51). Por outro lado, foi relatado em humanos que atividade física pesada, como corrida de alta intensidade, pode causar comprometimento da memória de trabalho e memória episódica, não sendo o mesmo evidente naquelas de média e baixa intensidade (52).

Assim, estudaremos o efeito da prática de atividade física (como caminhada e natação), em combinação com os estímulos imunes, sobre os processos de aprendizado I memorizaçáo na homeostase e após a malária experimental não grave por $P b A$ em camundongos $\mathrm{C} 57 \mathrm{BL} / 6$.

vi) Quais alteraçóes histopatológicas e imunológicas são detectadas imediatamente antes e em diferentes momentos após o tratamento e após os estímulos imunes posteriores a um episódio de malária não grave?

Estudos histopatológicos indicam alteração da permeabilidade e rompimento da BHE em decorrência do desenvolvimento da resposta imune inflamatória no sistema nervoso central em animais que desenvolvem MC (53). Em princípio, tal fenomenologia não seria observada em associação à $\mathrm{MnG}$ (11). Entretanto, os resultados de nosso grupo mostrando que um único episódio de $\mathrm{MnG}$ é capaz de causar danos cognitivos-comportamentais em camundongos C57BL/6 infectados com PbA (24) justificam o aprofundamento das investigaçóes nos cérebros dos animais para saber se, quando e de que forma o tecido nervoso é afetado/modulado mesmo por uma 
infecção de apenas quatro dias. Assim, análises histopatológicas estão em curso e estudos imunocitoquímicos e de expressão de genes de moléculas imunes serão feitos ao fim da infecção de quatro dias, e em diferentes momentos após o tratamento e após os estímulos imunes que se mostraram capazes de reverter tais déficits (40).

\section{vii) As alteraçóes cognitivo-comportamen-} tais estariam associadas a alteraçóes no padrão de expressáo gênica detectáveis no cérebro?

Distúrbios de comportamento, memória e aprendizado derivam de mudanças no padrão de expressão gênica de genes associados a essas características, e um mesmo conjunto gênico pode regular parâmetros neurocognitivos em camundongos e humanos.

Alguns genes já foram associados a alteraçóes de comportamento em animais. Marcadores associados à ansiedade, resposta a estresse e a via de recaptação de serotonina foram amplamente investigados. Uma vez que os genes não operam de forma independente, mas em função de um background genético e ambiental, tais estudos sugerem que essas alteraçóes não se devem a um ou outro gene específico atuando de forma isolada.

Estudos que avaliaram o comportamento de agressividade, se concentraram em alvos envolvendo a recaptação de serotonina e a enzima que metaboliza a norepinefrina, monoamino oxidase A (54). Em camundongos e humanos, genes associados à ansiedade também foram alvo de estudo e a via de recaptação de serotonina demonstrou desempenhar um papel importante na alteração de comportamento (55). Mais recentemente, genes de relógio biológico foram implicados como alvos importantes em transtornos de ansiedade (56). Genes associados à memória também já foram alvo de estudo (57) e representam ferramentas importantes na avaliação do prejuízo a aspectos comportamentais, de memória e de aprendizado em associados a parasitoses. Por esses motivos estamos buscando sinais de modificação no padrão de expressão gênica de marcadores associados a comportamento, memória e aprendizado em camundongos infectados, reforçando os resultados já obtidos em análises comportamentais em nosso laboratório.

Ainda que não exaustivo, o conjunto de perguntas citadas acima ilustra o repertório de projetos/estudos em andamento no LPM, IOC-Fiocruz, com os objetivos de contribuir para o melhor entendimento das alteraçóes neurocognitivas e comportamentais associadas à $\mathrm{MnG}$, e buscar modos de reverter os danos causados por ela.

\section{Conclusão}

Embora modelos animais possuam limitaçóes, eles correspondem à estratégia mais comumente utilizada atualmente para investigação de diferentes aspectos de doenças de 
caráter infeccioso como a malária, entre muitas outras. Além de ser de fácil manuseio e de custo relativamente baixo, como no caso de modelos experimentais murinos, eles podem ter impacto significativo no estudo da patogênese, inclusive de mecanismos envolvidos na geração de complicaçôes graves como a MC, já que apresentam muitas características similares aos achados da patologia humana. Exemplos são o comprometimento da BHE e a presença de sequelas cognitivas, ainda que após tratamento bem-sucedido com antimaláricos. $\mathrm{O}$ uso de modelo experimental murino em nosso grupo nos permitiu detectar déficits de memória e comportamento ansioso tardiamente após um episódio único de $\mathrm{MnG}(24,37)$, e demonstrar que estímulos imunes anti-inflamatórios, inclusive com vacinas humanas, podem reverter esses déficits (40). Para continuar investigando a evolução, a patologia, a expressão de genes e a resposta imune local associados aos danos provocados pela $\mathrm{MnG}$, assim como aprimorar os métodos para revertê-los, o uso de modelos experimentais com animais de laboratório se impóe.

\section{Agradecimentos}

BNSS, PRG, ABM, CCTLG, RMMAL \& LPS agradecem a CAPES, IOC, CNPqPibiti, CNPq-Pibic, Faperj INCT-NIM e Faperj Rede NI por suas bolsas de mestrado,
Pibiti, Pibic, doutorado, pós doutorado e pós doutorado, respectivamente. CTDR é Bolsista de Produtividade (CNPq) e Cientista do Nosso Estado (Faperj). O Laboratório de Pesquisa em Malária do Instituto Oswaldo Cruz da Fiocruz (LPM, IOC, Fiocruz) é um Laboratório Associado do Instituto Nacional de Ciência e Tecnologia em Neuroimunomodulação (INCT-NIM/Faperj/CNPq) e da Rede de Neuroinflamação (Rede-NEURIN/ Faperj). Os resultados e projetos citados no artigo tiveram ou têm apoio financeiro do POMIOC, Fiocruz e da Rede Temática da Faperj (processo SEI-260003/001169/2020) e estão autorizados pelos pareceres L-010/2015 e L-004/2020 da Comissão de Ética no Uso de Animais de laboratório (CEUA) do IOC, Fiocruz.

\section{REFERÊNCIAS BibLIOGRÁFICAS}

1. OMS - World Health Organization. World Malaria Report. 2020.

Disponível em:<https://www.who. int/teams/global-malaria-programme/ reports/world-malaria-report-2020> Acesso em 06/09/2021.

2. Sharp, PM, Plenderleith, LJ, Hahn, $\mathrm{BH}$ : Ape origins of human malaria. Annu. Rev. Microbiol. 2020; 74: 3963.

3. OPAS - Organização Pan Americana 
da Saúde/OMS. Malária. 2017.

Disponível em: <https://www.paho. $\mathrm{org} / \mathrm{pt} /$ topicos/malaria $>$ Acesso em 06/09/2021

4. White, NJ: Anaemia and malaria. Malar. J. 2018; 17(1): 1-17.

5. Martins, YC, Smith, MJ, PelajoMachado, M, Werneck, GL, Lenzi, HL, Daniel-Ribeiro, CT: Characterization of cerebral malaria in the outbred Swiss Webster mouse infected by Plasmodium berghei ANKA. Int. J. Exp. Pathol. 2009; 90(2): 119-130.

6. Khandare, AV, Bobade, D, Deval, M, Patil, T, Saha, B, Prakash, D: Expression of negative immune regulatory molecules, proinflammatory chemokine and cytokines in immunopathology of ECM developing mice. Acta Trop. 2017; 172: 58-63.

7. Ghazanfari, N, Mueller, SN, Heath, WR: Cerebral malaria in mouse and man. Front. immunol. 2018; 9: 2016.

8. Carvalho, LJM, Lenzi, HL, PelajoMachado, M, Oliveira, DN, DanielRibeiro, CT, Ferreira-da-Cruz, MF: Plasmodium berghei: cerebral malaria in CBA mice is not clearly related to plasma TNF levels or intensity of histopathological changes. Exp Parasitol. 2000; 95(1): 1-7.

9. Hee, L, Dinudom, A, Mitchell, AJ,
Grau, GE, Cook, DI, Hunt, NH, et al: Reduced activity of the epithelial sodium channel in malaria-induced pulmonary oedema in mice. Int. J. Parasitol. 2011; 41(1): 81-88.

10. Delahaye, NF, Coltel, N, Puthier, D, Flori, L, Houlgatte, R, Iraqi, FA, et al: Gene-expression profiling discriminates between cerebral malaria (CM)-susceptible mice and CMresistant mice. J. Infect. Dis. 2006; 193(2): 312-321.

11. Nacer, A, Movila, A, Baer, K, Mikolajczak, SA, Kappe, SH,Frevert, U: Neuroimmunological blood brain barrier opening in experimental cerebral malaria. PLoS Pathog. 2012; 8(10): e1002982.

12. Ball, EA, Sambo, MR., Martins, $M$, Trovoada, MJ, Benchimol, C, Costa, J, et al: IFNAR1 controls progression to cerebral malaria in children and CD8+ T cell brain pathology in Plasmodium berghei-infected mice. J. Immunol, 2013; 190(10): 5118-5127.

13. Bopp, SE, Rodrigo, E, González-Páez, GE, Frazer, M, Barnes, SW, Valim, C, et al: Identification of the Plasmodium berghei resistance locus 9 linked to survival on chromosome 9. Malar. J. 2013; 12(1): 1-12.

14. Strangward, P, Haley, MJ, Shaw, TN, Schwartz, JM, Greig, R, Mironov, A, et al: A quantitative brain map 
of experimental cerebral malaria pathology. PLoS Pathog. 2017; 13(3): e1006267.

15. Cabrales, P, Zanini, GM, Meays, D, Frangos, JA, \& Carvalho, LJ: Nitric oxide protection against murine cerebral malaria is associated with improved cerebral microcirculatory physiology. J. Infect. Dis. 2011; 203(10): 1454-1463.

16. Lima, MN, Oliveira, HA, Fagundes, PM, Estato, V, Silva, AY, Freitas, $\mathrm{RJ}$, et al: mesenchymal stromal cells protect against vascular damage and depression-like behavior in mice surviving cerebral malaria. Cytotherapy. 2021; 23(4): 25.

17. Cariaco, Y, Lima, WR, Sousa, R, Nascimento, LAC, Briceño, MP, Fotoran, WL, et al: Ethanolic extract of the fungus Trichoderma stromaticum decreases inflammation and ameliorates experimental cerebral malaria in C57BL/6 mice. Sci. Rep. 2018; 8(1): 1-15.

18. John, CC, Bangirana, P, Byarugaba, J, Opoka, RO, Idro, R, Jurek, AM, et al: Cerebral malaria in children is associated with long-term cognitive impairment. Pediatrics. 2008; 122(1): e92-e99.

19. Freeman, BD, Martins, YC, AkideNdunge, OB, Bruno, FP, Wang, $\mathrm{H}$, Tanowitz, HB, et al: Endothelin-1 mediates brain microvascular dysfunction leading to long-term cognitive impairment in a model of experimental cerebral malaria. PLoS Pathog. 2016; 12(3): e1005477.

20. Lacerda-Queiroz, N, Teixeira, MM, Teixeira, AL: Imunopatogênese da malária cerebral. Ver Bras Neurol. 2008; 44(1): 13-9.

21. Fernando, SD., Gunawardena, DM, Bandara, MRSS, De Silva, D, Carter, $\mathrm{R}$, Mendis, KN, et al: The impact of repeated malaria attacks on the school performance of children. Am J TropMedHyg. 2003; 69(6): 582-588.

22. Tapajós, R, Castro, D, Melo, G, Balogun, S, James, M, Pessoa, R, et al: Malaria impact on cognitive function of children in a peri-urban community in the Brazilian Amazon. Malar. J. 2019; 18(1): 1-12.

23. Reis, PA, Comim, CM, Hermani, F, Silva, B, Barichello, T, Portella, A, et al: Cognitive dysfunction is sustained after rescue therapy in experimental cerebral malaria, and is reduced by additive antioxidant therapy. PLoS Pathog. 2010; 6(6): e1000963.

24. de Sousa, LP, de Almeida, RF, RibeiroGomes, FL, de Moura Carvalho, LJ, e Souza, TM, de Souza, DOG, et al: Long-term effect of uncomplicated Plasmodium berghei ANKA malaria on memory and anxiety-like behaviour in 
C57BL/6 mice. Parasit Vectors. 2018; 11(1): 1-9.

25. Schiller, M, Ben-Shaanan, TL, Rolls, A: Neuronal regulation of immunity: why, how and where?. Nat Rev Immunol. 2021; 21(1): 20-36.

26. Li, C, Seixas, E, Langhorne, J: Rodent malarias: the mouse as a model for understanding immune responses and pathology induced by the erythrocytic stages of the parasite. Med Microbiol Immunol, 2001; 189(3): 115-126.

27. Curfs, JH., Schetters, TP, Hermsen, CC., Jerusalem, CR, Van Zon, AA, Eling, WM: Immunological aspects of cerebral lesions in murine malaria. Clin. Exp. Immunol. 1989; 75(1): 136.

28. Rest, JR: Cerebral malaria in inbred mice. I. A new model and its pathology. Trans. R. Soc. Trop. Med. Hyg. 1982; 76(3): 410-415.

29. de Kossodo, S, Grau, GE: Profiles of cytokine production in relation with susceptibility to cerebral malaria. J. Immunol. 1993; 151(9): 4811-4820.

30. Neill, AL, Hunt, NH: Pathology of fatal and resolving Plasmodium berghei cerebral malaria in mice. Parasitology. 1992; 105(2): 165-175.

31. Kamiyama, T, Tatsumi, M, Matsubara, J, Yamamoto, K, Rubio, Z, Cortes, $\mathrm{G}$ et al: Manifestation of cerebral malaria-like symptoms in the WM/Ms rat infected with Plasmodium berghei strain NK65. J. Parasitol. 1987; 11381145.

32. Lacerda-Queiroz, N, Lima OC, Carneiro CM, Vilela MC, Teixeira AL, Teixeira-Carvalho A, et al: Plasmodium berghei NK65 induces cerebral leukocyterecruitment in vivo: na intravitalmicroscopicstudy. Acta Trop. 2011; 120(1-2): 31-9.

33. Haque, A, Echchannaoui, H, Seguin, R, Schwartzman, J, Kasper, LH, Haque, S: Cerebral malaria in mice: interleukin-2 treatment induces accumulation of $\gamma \delta \mathrm{T}$ cells in the brain and alters resistant mice to susceptiblelike phenotype. Am. J. Pathol. 2001; 158(1): 163-172.

34. Kaul, DK, Nagel, RL, Llena, JF, Shear, HL: Cerebral malaria in mice: demonstration of cytoadherence of infected red blood cells and microrheologic correlates. Am. J. Trop. Med. Hyg. 1994; 50(4): 512-521.

35. Guha, SK, Tillu, R, Sood, A, Patgaonkar, M, Nanavaty, IN, Sengupta, A, et al: Single episode of mild murine malaria induces neuroinflammation, alters microglial profile, impairs adult neurogenesis, and causes deficits in social and anxiety-like behavior. Brain Behav. Immun.2014; 42: 123-137.

36. Potter, $\mathrm{S}$, Chan-Ling, T, Ball, HJ, 
Mansour, H, Mitchell, A, Maluish, L et al: Perforin mediated apoptosis of cerebral microvascular endothelial cells during experimental cerebral malaria. Int J Parasitol. 2006; 36(4): 485-496.

37. Rosa-Gonçalves, P: Dinâmica e imunomodulação de déficits cognitivos e alteraçôes comportamentais da malária não grave experimental. Dissertação (Mestrado em Biologia Parasitária).Rio de Janeiro: Instituto Oswaldo Cruz, Fiocruz. 2021.

38. Dutta, S, Sengupta, P: Men and mice: relating their ages. Life Sci. 2016; 152: 244-248.

39. Nataf, S: Autoimmunity as a driving force of cognitive evolution. Front Neurol . 2017; 11: 582.

40. de Sousa, LP, Ribeiro-Gomes, FL, de Almeida, RF, e Souza, TM, Werneck, GL, Souza, DO et al: Immune system challenge improves recognition memory and reverses malaria-induced cognitive impairment in mice. Sci. Rep. 2021; 11(1): 1-11.

41. Dayananda KK, Achur RN, Gowda DC: Epidemiology, drug resistance, and pathophysiology of Plasmodium vivax malaria. J Vector Borne Dis. 2018; 55(1):1-8.

42. American College of Sports Medicine Position Stand American Heart Association. 1998; 2007.

43. Walsh, NP, Gleeson, M, Pyne, DB,
Nieman, DC, Dhabhar, FS, Shephard, RJ, et al: Position statement part two: maintaining immune health. Exerc. Immunol. Rev. 2011; 17: 64-103.

44. Pedersen, BK, Hoffman-Goetz, L: Exercise and the imune system: regulation, integration, and adaptation. Physiol. 2000; 80: 10551081.

45. Lili T, Cheng, P: Alterations of immunoendocrine responses during the recovery period after acute prolonged cycling. Eur. J. Appl. Physiol. 2007; 101: 539-546.

46. Voss, MW, Weng, TB, NarayanaKumanan, K, Cole, RC, Wharff, C, Reist, L et al: Acute exercise effects predict training change in cognition and connectivity. Med Sci Sports Exerc. 2020; 52(1): 131.

47. Ji, Z, Feng, T, Mei, L, Li, A, Zhang, C: Influence of acute combined physical and cognitive exercise on cognitive function: an NIRS study. PeerJ. 2019; 7: e7418.

48. Moreau, D, Chou, E: The acute effect of high-intensity exercise on executive function: a meta-analysis. Perspect. Psychol. Sci. 2019; 14(5): 734-764.

49. Abasiyanik Z, Ertekin O, Kahraman T, Yigit P, Ozakbas S: The effects of Clinical Pilates training on walking, balance, fall risk, respiratory, and cognitive functions in persons with 
multiple sclerosis: A randomized controlled trial. Explore. 2019; 1-9.

50. Lin, XY, Cui, Y, Wang, L, \& Chen, W: Chronic exercise buffers the cognitive dysfunction and decreases the susceptibility to seizures in PTZtreated rats. Epilepsy Behav. 2019; 98: 173-187.

51. Lourenco, MV, Frozza, RL, de Freitas, GB, Zhang, H, Kincheski, GC, Ribeiro, FC, et al: Exerciselinked FNDC5/irisin rescues synaptic plasticity and memory defects in Alzheimer's models. Nat. Med. 2019; 25(1): 165-175.

52. Loprinzi, PD, Harris, F, McRaney, K, Chism, M, Deming, R, Jones, $\mathrm{T}$, et al: Effects of acute exercise and learning strategy implementation on memory function. Medicina. 2019; 55(9): 568.

53. De Souza, JB, Riley, EM: Cerebral malaria: the contribution of studies in animal models to our understanding of immunopathogenesis. MicrobInfect. 2002; 4(3): 291-300.

54. Craig, IW, Halton, KE: Genetics of human aggressive behaviour. Hum. Genet. 2009; 126(1): 101-113.

55. Hovatta, I, Barlow, C: Molecular genetics of anxiety in mice and men. Ann. Med. 2008; 40(2): 92-109.

56. Buoli, M, Serati, M, Grassi, S, Pergoli, L, Cantone, L, Altamura, AC, et al:The role of clock genes in the etiology of Major Depressive Disorder: Special Section on "Translational and Neuroscience Studies in Affective Disorders". Section Editor, Maria Nobile MD, PhD. This Section of JAD focuses on the relevance of translational and neuroscience studies in providing a better understanding of the neural basis of affective disorders. The main aim is to briefly summaries relevant research findings in clinical neuroscience with particular regards to specific innovative topics in mood and anxiety disorders. J Affect Disord. 2018; 234: 351-357.

57. Kandel, ER: The molecular biology of memory: cAMP, PKA, CRE, CREB1, CREB-2, and CPEB. Mol Brain. 2012; 5(1): 1-12. 